\title{
Coque de petróleo como controlador de filtrado en fluidos de perforación base aceite
}

\section{(Petroleum coke as a filter controller in oil-base drilling fluid)}

\author{
Rubén Vega ${ }^{1}$, Martín León ${ }^{1}$, Patricia Lara ${ }^{1}$
}

\begin{abstract}
Resumen:
La gran cantidad de coque de petróleo producido en Venezuela, conllevó a su uso como controlador de filtrado en fluidos base aceite. Inicialmente el coque se sometió a un proceso de extracción de aceite mediante el método Soxhlet y extracción de azufre mediante microondas. Seguidamente se le realizó una Espectroscopia de Infrarrojo (IR) y se comparó con otra espectroscopia del coque de petróleo natural, donde quedó evidenciado la efectividad del proceso aplicado. Se formularon cuatro (4) fluidos de perforación (12 lpg) con coque como controlador de filtrado, variando su concentración en $6,8,10$ y $12 \mathrm{lb} / \mathrm{bl}$ y después de un envejecimiento dinámico $\left(300^{\circ} \mathrm{F}\right.$, $100 \mathrm{lpcm}$ ) se estudiaron las propiedades físicas de viscosidad plástica, punto cedente, gel $10 \mathrm{~s}$, gel 10 min y filtrado, obteniéndose rango de valores de 25,3-26,3; 5,7-6,3; 4-5; 7-10 y 10,3-10,9 respectivamente. Un estudio estadístico de parcelas divididas demostró que existieron diferencias estadísticas significativas entre las propiedades físicas de los fluidos formulados y las propiedades físicas de un fluido patrón con lignito como controlador de filtrado. Se concluyó que a pesar de las diferencias estadísticas presentadas es posible el uso de coque de petróleo como controlador de filtrado para las condiciones en estudio.
\end{abstract}

Palabras clave: lignito, método Soxhlet, Espectroscopia IR, propiedades físicas, control de filtrado.

\begin{abstract}
:
The large amount of petroleum coke produced in Venezuela, leadsed to its use as a filtering controller in oil-based fluids. Initially, the coke was subjected to an oil extraction process using the Soxhlet method and extraction of sulfur by means of microwaves. Then an infrared Spectroscopy (IR) was performed and compared with another spectroscopy of natural petroleum coke, where the effectiveness of the applied process was evidenced. Were made, four (4) drilling fluid (12 ppg) with coke as filter driver, varying its concentration in $6,8,10$ and $12 \mathrm{lb} / \mathrm{bl}$ and after dynamic aging $\left(300^{\circ} \mathrm{F}, 100 \mathrm{lpcm}\right)$ the physical properties of plastic viscosity, yield point, gel 10 $\mathrm{s}$, gel $10 \mathrm{~min}$ and filtrate were studied, obtaining a range of values of 25.3-26.3; 5.7$6.3 ; 4-5 ; 7-10$ and $10.3-10.9$ respectively. A statistical study of split plots demonstrated that there were significant statistical differences between the physical properties of the formulated fluids and the physical properties of a standard fluid with lignite as a filtering controller. It was concluded that despite the statistical differences presented, the use of petroleum coke as a filter controller for the conditions under study is possible.
\end{abstract}

Keywords: lignite, Soxhlet method, spectroscopy (IR), physical properties, filtering controller

\footnotetext{
1 Universidad de Oriente, Maturín, Venezuela (rvegas@udo.edu.ve, \{M1C9DB1E, M1D9451F\} @alum.udo.edu.ve )
} 


\section{Introducción}

Con la perforación de pozos de petróleo se persigue la construcción de un hoyo que comunique la superficie con el yacimiento y por ende corroborar la existencia de crudo. Este proceso emplea un equipo rotatorio y fluidos de perforación los cuales facilitan el trabajo. La composición de los fluidos varía según las exigencias del pozo, las capacidades de los equipos y los riesgos ambientales. Los ingenieros formulan los fluidos de perforación para controlar las presiones del subsuelo, disminuir el daño a la formación, minimizar la posibilidad de pérdida de circulación, controlar la erosión del pozo y optimizar los parámetros de operación, tales como taza de perforación y limpieza del hoyo (Wiliamson, 2013).

En sus inicios el fluido empleado era una mezcla de agua con arcilla pero no permitía el control de algunas propiedades a medida que se incrementaba la profundidad de perforación. Es por ello, que a partir de la segunda mitad del siglo XX se desarrollan los fluidos base aceite, que mejoran dichas propiedades, no reaccionan con las arcillas y favorecen la tasa de penetración así como su efectividad (López, 2003). En comparación con la mayoría de los fluidos base agua, los fluidos base aceite poseen una mayor estabilidad de la viscosidad, mayor tolerancia térmica y mejores propiedades de inhibición, lo que los convierte en la solución preferida para las operaciones de alta presión y altas temperaturas (HPHT) (Faergestad y Stachan, 2014). No obstante, los fluidos base aceite, representan elevados costos en las operaciones de perforación, entre el 20 al $25 \%$ del costo total del pozo (Vega et al., 2016).

Para lograr las funciones del fluido de perforación, a la fase líquida o fase continua es necesario agregarle ciertos componentes para mejorarle las propiedades físicas, llamados aditivos, entre los cuales se pueden mencionar agentes densificantes, viscosificantes, humectantes, controladores de filtrado entre otros. La variación de tipo, calidad y cantidad de cualquiera de los aditivos empleados conlleva a un cambio de las propiedades físicas y sobre todo reológicas. Las propiedades reológicas de estos fluidos deben ser evaluadas y controladas con la finalidad que realicen, en forma óptima y económica, algunas tareas principales como: (a) la lubricación de la barrena; (b) el enfriamiento de la tubería de perforación; y (c) el transporte de los recortes de la formación hacia la superficie, en donde la viscosidad del fluido se considera un factor importante para mantener suspendidos los recortes de perforación (Gómez et al., 2009).

Cuando el fluido es enviado al fondo del hoyo tiene contacto con algunas formaciones de roca que son porosas y permeables y actúan como un filtro que permite el paso de una fracción de la fase líquida, fenómeno denominado pérdida de filtrado (Ariza et al., 2013). El aditivo para disminuir esta pérdida es denominado controlador de filtrado. Con respecto a dicho agregado en los fluidos base aceite se suele emplear lignito, lignosulfonato o carbonato de calcio. Un lignito es un carbón mineral que se forma por compresión, es de color negro o pardo y su concentración en carbono varía entre el $60 \%$ y el $75 \%$ (CIED-PDVSA, 2002)

En otro orden de ideas, las acumulaciones de crudos pesados y extrapesados han adquirido bastante importancia en las últimas décadas, ya que las compañías operadoras de petróleo han tenido que acudir a la explotación de yacimientos no convencionales de petróleo, para completar la producción que se demanda mundialmente (Lowy, Garrido y Melo, 2015). Venezuela cuenta con las mayores reservas de crudos pesados y extrapesados del mundo, las cuales se estiman en más de 235 millardos de barriles de petróleo (Petróleos de Venezuela S.A, 2006)

Como consecuencia de la explotación de este tipo de crudo, el país también cuenta con grandes cantidades de coque de petróleo; se estima que solo el Complejo Petroquímico General de División José Antonio Anzoátegui produce 20.000 toneladas diarias de coque de petróleo (MPPEUCT, 2012). El coque de petróleo es un producto residual de elevado contenido de carbono, resultante de la pirolisis de las fracciones pesadas obtenidas en el refino de petróleo, que ha pasado de un estado líquido a 
cristalino durante el proceso de carbonización y que está constituido por carbono no grafítico pero grafitizable, el cual tiene un alto poder calorífico y un porcentaje de carbono superior al 90\% (Gorrin, Basantas y López J, 2011). El coque de petróleo se utiliza como materia prima para la obtención de carbono y como combustible para la generación de calor (Katz, 2001). Su composición es básicamente carbón, cenizas y azufre (Da Silva et al., 2004).

Ante lo descrito, la presente investigación tuvo como propósito corroborar si el coque de petróleo del Complejo Petroquímico General de División José Antonio Anzoátegui, Venezuela actúa como controlador de filtrado en fluidos de perforación base aceite mineral Vassa en sustitución de un lignito organofílico comercial.

\section{Metodología}

La investigación se desarrolló en el laboratorio de fluidos de perforación de la Universidad de Oriente, Núcleo de Monagas, Venezuela. Así, inicialmente se procedió a extraerle el aceite contenido en el coque de petróleo mediante la aplicación del método Soxhlet aplicando la norma EPA 3540C (2015), para ello se empleó Xileno como disolvente. A continuación se conoció el contenido de azufre en el coque de petróleo aplicando el procedimiento descrito en la norma ASTM 3177-07 y se disminuyó su contenido mediante la aplicación del procedimiento descrito por Kraüter et al.; (2003). Seguidamente al coque de petróleo tratado (CPT) se le realizó una espectroscopia de Infrarrojo (IR) empleando para ello un equipo Perkin Elmer FTIR 1600, el cual permitió generar espectros a partir de una pastilla de Bromuro de Potasio $(\mathrm{KBr})$ elaborada con el material en estudio.

Posteriormente, el CPT se trabajó para hacerlo organofílico (Pinzón, 2006) iniciando de una dispersión de este en agua destilada, a la cual se le añadió una solución de amina cuaternaria (Dodigen 226) y se agitó durante 30 min. A continuación, a partir de una formulación para un fluido de perforación base aceite de densidad de 12 lpg (Samán Tecnología Integral en Petróleo, C.A ,2010) (Tabla 1) se propusieron 4 fluidos variando únicamente la concentración de coque de petróleo en 6, 8, 10 y $12 \mathrm{lb} / \mathrm{bl}$ ( Tabla 2).

Cabe destacar que la máxima cantidad de controlador de filtrado que se debe emplear para este tipo de fluido es de $8 \mathrm{lb} / \mathrm{bl}$, por tratarse de una emulsión inversa con $70 \%$ aceite y $30 \%$ agua, además de poseer $\mathrm{CaCl}_{2}$ al $30 \%$ (Samán Tecnología Integral en Petróleo, C.A ,2010). Como el objetivo de la investigación era conocer si el coque de petróleo puede ser empleado como controlador de filtrado se decidió emplear $12 \mathrm{lb} / \mathrm{bl}$ como máximo.

Tabla 1. Formulación del fluido de perforación base aceite

\begin{tabular}{llll}
\hline Producto & Concentración & Unidad & Mezcla (min) \\
\hline Aceite Vassa & 237 & $\mathrm{~cm}^{3}$ & - \\
\hline Cal hidratada & 7 & $\mathrm{~g}$ & 5 \\
\hline Emulsificante & 8 & $\mathrm{~cm}^{3}$ & 10 \\
\hline Controlador de filtrado & - & $\mathrm{g}$ & 15 \\
\hline CaCl 2 al 30\% & 59 & $\mathrm{~cm}^{3}$ & 30 \\
\hline Humectante & 8 & $\mathrm{~cm}^{3}$ & 5 \\
\hline Arcilla organofílica & 12 & $\mathrm{~g}$ & 10 \\
\hline Barita & 229 & $\mathrm{~g}$ & 15 \\
\hline
\end{tabular}

Tabla 2. Fluidos y concentración de coque y lignito empleado

\begin{tabular}{lll}
\hline Fluido & & Concentración $(\mathbf{l b} / \mathbf{b l})$ \\
\hline \multirow{3}{*}{ Coque } & FA & 6 \\
\cline { 2 - 3 } & FB & 8 \\
\cline { 2 - 3 } & FC & 10 \\
\cline { 2 - 3 } & FD & 12 \\
\hline Patrón (lignito) & & 8 \\
\hline
\end{tabular}


Formulados los fluidos de perforación y posterior a un proceso de envejecimiento dinámico con condiciones de temperatura de $300^{\circ} \mathrm{F}$, presión de $100 \mathrm{lpcm}$ y rotación de 100 rpm durante 16 horas que simularon las condiciones de pozos, se les midieron a los fluidos las propiedades físicas de estabilidad eléctrica, viscosidad plástica (VP), punto cedente (PC), esfuerzo gel (EG) y filtrado AT-AP (F) de acuerdo con la norma API RP 13B-2 (2003). Bajo las mismas condiciones se formuló y envejeció un fluido de perforación base aceite patrón $(\mathrm{P})$ con controlador de filtrado comercial (lignito) de 8 $\mathrm{lb} / \mathrm{bbl}$, al cual también se le midieron las propiedades físicas mencionadas.

Finalmente, mediante el uso de un diseño factorial aleatorio, se aplicó un estudio estadístico de parcelas divididas de acuerdo con la metodología planteada por Little y Hills, (1998); la misma permitió comparar el efecto de la concentración de coque en los fluidos propuestos y el fluido patrón. También se aplicó el método de diferencias medias significativas (DMS) para establecer diferencias entre las distintas propiedades de los fluidos, para ello se utilizó una hoja de Excel.

\section{Resultados y discusión}

La cantidad de azufre inicialmente presente en la muestra de coque era de 5,49\% y una vez aplicado el proceso de desulfurización se logró reducirlo a 2,28\%. Luego se trató el coque de petróleo con Xileno mediante el método Soxhlet donde se extrajo el aceite contenido en él (19\%). Posterior a los procesos descritos se observa en la espectroscopia obtenida (Figura 1) que en el intervalo entre $3300-3700 \mathrm{~cm}^{-1}$ corresponde a agua o grupos $\mathrm{OH}$ que no son representativos en el estudio realizado. Entre $3000-3100 \mathrm{~cm}^{-1} \mathrm{se}$ percibe la presencia de una de zona aromáticos $\mathrm{C}-\mathrm{H}$; en el intervalo $2800-3000 \mathrm{~cm}^{-1}$ se muestra la presencia de zona de alifáticos $\mathrm{C}-\mathrm{H}$. En el intervalo $1680-1790 \mathrm{~cm}^{-1}$ marca la presencia de $\mathrm{C}=\mathrm{O}$ de zona carboxílicos, casi despreciable compuesto básicamente por ésteres. El intervalo de $1500-1680 \mathrm{~cm}^{-1}$ representa la zona característica de enlaces $\mathrm{C}=\mathrm{C}$ propios de coque.

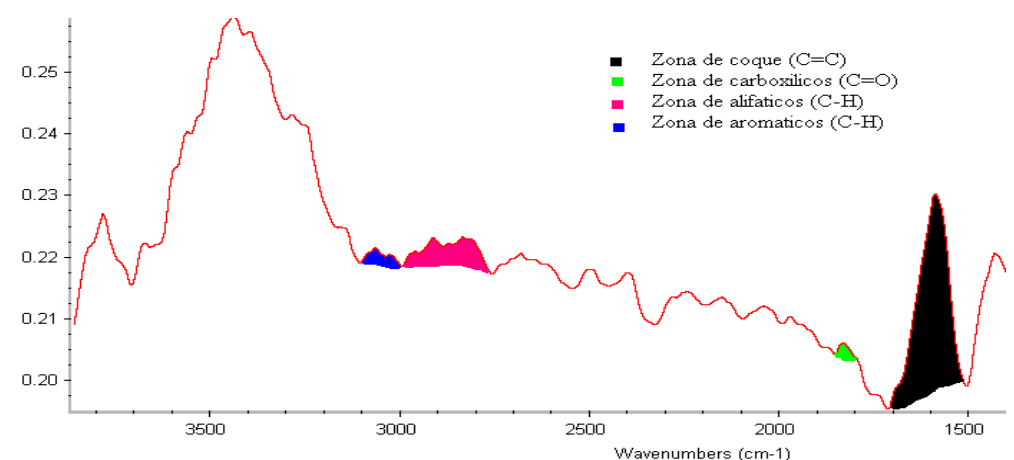

Figura 1.Espectroscopia (IR) del coque tratado con Xileno

En contraste con la espectroscopia obtenida por Vega et al.; (2016) (Figura 2), se observa que con el proceso de lavado con Xileno disminuyó considerablemente la cantidad de alifáticos y carboxílicos, demostrando que el Xileno tiene una capacidad de arrastre de componentes presentes en el coque de petróleo natural y este método garantiza la obtención de un coque limpio o puro.

Una vez tratado el coque de petróleo con una amina cuaternaria se aplicó una prueba cualitativa de suspensión (Figura 3), comúnmente empleada en la industria petrolera para conocer qué tan organofílico es el lignito comercial con el aceite Vassa. Se observa en la Figura 3 (a), que el coque sin tratamiento decanta en el aceite Vassa, mientras que para el mismo tiempo el coque de petróleo tratado (Figura 3 (b)), se mantiene suspendido en el aceite, por lo que se puede inferir que el coque de petróleo se transformó en organofílico. 


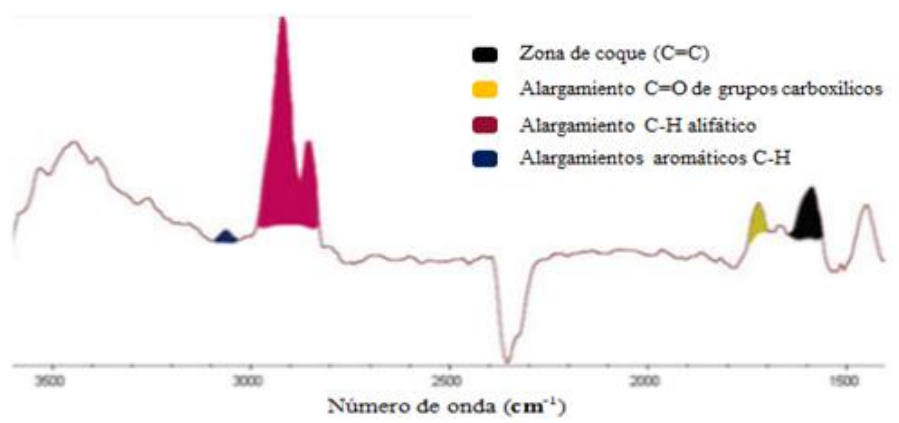

Figura 2.Espectroscopia (IR) del coque no tratado, natural

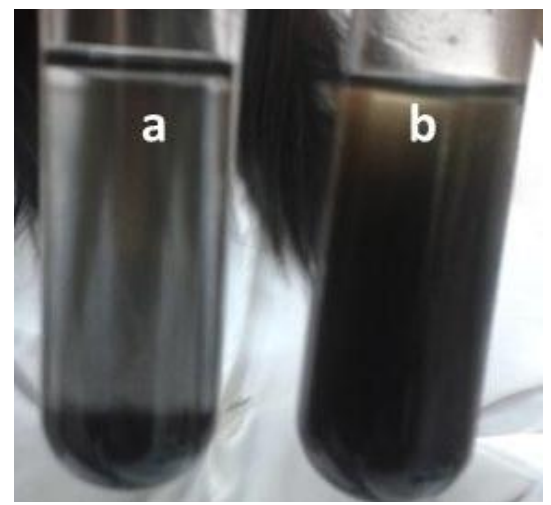

Figura 3. Prueba cualitativa de transformación de coque de petróleo en organofílico

Concernientes a las propiedades físicas de los fluidos en la Tabla 3 se muestran los valores promedios de las mismas después del envejecimiento dinámico. La densidad debió corresponder a 12 lpg, pero se nota una disminución en décimas con la adición de las distintas concentraciones de coque de petróleo. Cabe destacar que durante el estudio se observó que la barita no se sedimentó, lo que es un aspecto importante en la formulación dado que indica que el fluido tiene la capacidad de mantener suspendidos los sólidos y que tan exacto fue el balance de masa en las formulaciones de los fluidos. De manera general la utilización de este coque de petróleo tratado no afectó directamente las variaciones de densidad de los fluidos ni genera una desviación representativa del valor correspondiente.

Tabla 3. Valores promedios de las propiedades físicas en estudio

\begin{tabular}{|c|c|c|c|c|c|c|c|}
\hline \multirow{3}{*}{ Fluido } & \multicolumn{7}{|c|}{ Propiedades físicas } \\
\hline & \multirow{2}{*}{$\begin{array}{l}\text { Densidad } \\
\text { (lpg) }\end{array}$} & \multirow{2}{*}{$\begin{array}{l}\text { Viscosidad } \\
\text { plástica } \\
\text { (cP) }\end{array}$} & \multirow{2}{*}{$\begin{array}{l}\text { Punto } \\
\text { cedente } \\
\text { (lb/100pie }^{2} \text { ) }\end{array}$} & $\begin{array}{l}\text { Esfuerzo } \\
\left(\mathrm{lb} / 100 \mathrm{pie}^{2}\right)\end{array}$ & gel & \multirow{2}{*}{$\begin{array}{l}\text { Filtrado } \\
\text { (mL/30min) }\end{array}$} & \multirow{2}{*}{$\begin{array}{l}\text { Estabilidad } \\
\text { Eléctrica } \\
\text { (Volt) }\end{array}$} \\
\hline & & & & $10 \mathrm{~s}$ & $\begin{array}{l}10 \\
\min \end{array}$ & & \\
\hline FA & 11,7 & 25,3 & 6,0 & 4 & 7 & 10,9 & 1050 \\
\hline FB & 11,8 & 26,3 & 5,7 & 5 & 10 & 10,7 & 1100 \\
\hline $\mathrm{FC}$ & 11,7 & 25,3 & 5,7 & 4 & 10 & 10,6 & 1100 \\
\hline FD & 11,8 & 26,3 & 6,3 & 5 & 9 & 10,3 & 1175 \\
\hline
\end{tabular}

El valor de la viscosidad plástica no debe exceder en ningún caso el doble del peso del fluido y siempre debe mantenerse en los niveles más bajos posible (Prieto, 2007); para el caso en estudio se debería tener un valor aproximado a $24 \mathrm{cP}$. En la Tabla 3, se detalla que de los sistemas formulados los que más se acercan a este valor son los sistemas A y C. Para las diferentes concentraciones la viscosidad plástica fue superior sin presentar variación representativa entre los tratamientos, demostrando que al aumentar la concentración de sólidos aumenta la viscosidad plástica. 
En los fluidos formulados se observó un bajo punto cedente debido a que las fuerzas de atracción son mínimas, lo cual no es lo idóneo. Se puede inferir que el bajo punto cedente se debió a que el humectante agregado evitó que se creara la interacción entre los aditivos del sistema debido a que este actúa creando una película de aceite en los sólidos haciéndolos preferiblemente humedecidos por aceite.

Respecto a la tixotropía de los fluidos, los cuatro sistemas formulados generaron geles después del envejecimiento en el rango de $4-5 \mathrm{lb} / 100$ pie $^{2}$ para los 10 segundos y un rango de 7-10 lb/100 pie $^{2}$ para los 10 minutos, encontrándose entre el rango de los geles favorables, presentando un valor bajo a los 10 segundos y un valor mediano a los 10 minutos.

Tabla 4. Fluidos empleados, organización por parcela principal, bloques y tratamientos

\begin{tabular}{|c|c|c|c|c|c|c|}
\hline \multirow{2}{*}{ Fluido } & & \multicolumn{3}{|c|}{ Bloques } & \multicolumn{2}{|l|}{ Tratamientos } \\
\hline & & $\mathbf{I}$ & II & III & Totales (Tt) & Medias (Xt) \\
\hline \multirow{5}{*}{ Patrón } & Viscosidad plástica (VP) & 35 & 35 & 34 & 104 & 34,67 \\
\hline & Punto cedente (PC) & 6 & 6 & 7 & 19 & 6,33 \\
\hline & Gel 10s & 9 & 8 & 9 & 26 & 8,67 \\
\hline & Gel 10min & 17 & 15 & 15 & 47 & 15,67 \\
\hline & Filtrado & 6 & 7 & 6 & 19 & 6,33 \\
\hline \multicolumn{2}{|c|}{ Totales de las parcelas $\left(T p p_{1}\right)$} & 73 & 71 & 71 & 215 & 14,33 \\
\hline \multirow{5}{*}{$\begin{array}{l}\text { FA } \\
(6 \mathrm{lpb})\end{array}$} & Viscosidad plástica (VP) & 26 & 25 & 25 & 76 & 25,33 \\
\hline & Punto cedente (PC) & 6 & 6 & 6 & 18 & 6,00 \\
\hline & Gel 10s & 4 & 4 & 5 & 13 & 4,33 \\
\hline & Gel 10min & 7 & 8 & 7 & 22 & 7,33 \\
\hline & Filtrado & 10,8 & 10,6 & 10,6 & 32 & 10,67 \\
\hline \multicolumn{2}{|c|}{ Totales de las parcelas $\left(\mathrm{Tpp}_{2}\right)$} & 53,8 & 53,6 & 53,6 & 161 & 10,73 \\
\hline \multirow{5}{*}{$\begin{array}{l}\text { FB } \\
(8 \mathrm{Ipb})\end{array}$} & Viscosidad plástica (VP) & 27 & 26 & 26 & 79 & 26,33 \\
\hline & Punto cedente (PC) & 5 & 6 & 6 & 17 & 5,67 \\
\hline & Gel 10s & 4 & 5 & 5 & 14 & 4,67 \\
\hline & Gel 10min & 10 & 9 & 10 & 29 & 9,67 \\
\hline & Filtrado & 10,8 & 10,6 & 10,8 & 32,2 & 10,73 \\
\hline \multicolumn{2}{|c|}{ Totales de las parcelas $\left(\mathrm{Tpp}_{3}\right)$} & 56,8 & 56,6 & 57,8 & 171,2 & 11,41 \\
\hline \multirow{5}{*}{$\begin{array}{l}\text { FC } \\
(10 \mathrm{lpb})\end{array}$} & Viscosidad plástica (VP) & 25 & 26 & 26 & 77 & 25,67 \\
\hline & Punto cedente (PC) & 5 & 6 & 6 & 17 & 5,67 \\
\hline & Gel 10s & 4 & 4 & 5 & 13 & 4,33 \\
\hline & Gel 10min & 10 & 10 & 9 & 29 & 9,67 \\
\hline & Filtrado & 10,6 & 10,6 & 10,7 & 31,9 & 10,63 \\
\hline \multicolumn{2}{|c|}{ Totales de las parcelas $\left(\mathrm{Tpp}_{4}\right)$} & 54,6 & 56,6 & 56,7 & 167,9 & 11,19 \\
\hline \multirow{5}{*}{$\begin{array}{l}\text { FD } \\
(12 \mathrm{lpb})\end{array}$} & Viscosidad plástica (VP) & 26 & 26 & 27 & 79 & 26,33 \\
\hline & Punto cedente (PC) & 6 & 7 & 6 & 19 & 6,33 \\
\hline & Gel 10s & 5 & 4 & 5 & 14 & 4,67 \\
\hline & Gel 10min & 10 & 9 & 9 & 28 & 9,33 \\
\hline & Filtrado & 10,4 & 10,2 & 10,4 & 31 & 10,33 \\
\hline \multicolumn{2}{|c|}{ Totales de las parcelas ( $\left.\mathrm{Tpp}_{5}\right)$} & 57,4 & 56,2 & 57,4 & 171 & 11,40 \\
\hline \multicolumn{2}{|c|}{ Totales de los bloques (Tbl) } & 295,6 & 294 & 296,5 & 886,10 & 11,81 \\
\hline
\end{tabular}


En los resultados obtenidos de la prensa (AT-AP) no se presentan diferencias apreciables en el volumen de filtrado entre los fluidos formulados. Se puede decir que se obtuvieron volúmenes de filtrado parecidos en los diferentes tratamientos, de unas décimas por encima del valor máximo fijado (10 mL) por (MI-SWACO, 2001). Para los fluidos de perforación base aceite hubo una mayor filtración de la esperada pero no representativa con respecto al valor máximo fijado, tomando en cuenta que el coque no está diseñado para el control de filtrado como los aditivos ya formulados para este fin.

Finalmente la estabilidad eléctrica presentada por los fluidos evidenció resultados aceptables porque los mismos contaron dentro de su formulación con $\mathrm{CaCl}_{2}$ al $30 \%$ el cual es un conductor y disminuye la resistividad pero no afecta la emulsión. Cabe destacar que una emulsión es más estable en la medida que las gotas de agua se hacen más pequeñas, uniformes y los sólidos agregados o incorporados se mantienen humectados por aceite. El agua genera viscosidad, resistencia de gel y contribuye con el controlar del filtrado porque las gotas se comportan como sólidos suspendidos (Prieto, 2007); quizás ello haya contribuido a la obtención de los valores de filtrado mostrados.

Concerniente al estudio estadístico, en la Tabla 4 se muestra la organización por parcela principal, bloques y tratamientos del estudio de parcelas divididas. En la Tabla 5 se ven los valores totales y promedios de las propiedades necesarios para el desarrollo del método.

Tabla 5. Propiedades de los fluidos

\begin{tabular}{llllll}
\hline \multicolumn{1}{c}{ Propiedades } & & & & \\
\hline & Viscosidad plática & Punto cedente & Gel 10s & Gel 10min & Filtrado \\
\hline Totales & 415 & 90 & 80 & 155 & 146,10 \\
\hline Promedio & 27,67 & 6 & 5,33 & 10,33 & 9,74 \\
\hline
\end{tabular}

Una vez establecido el diseño de parcelas dividas se desarrolló el análisis de varianza (Tabla 6), se detalla que el valor del $F_{\text {observado }}(47,92)$ de Fluidos x Propiedades es mayor que el $F_{\text {tabulado }}(1,90)$, lo que indica una diferencia altamente significativa entre los fluidos y sus propiedades, es decir que las propiedades de los fluidos con coque como controlador de filtrado no presentan un comportamiento semejante a las propiedades del fluido patrón con lignito.

Tabla 6. Análisis de varianza. Experimento de los fluidos por propiedades

\begin{tabular}{llllll}
\hline & gl & SC & CM & F (Obs) & F tabla (5\%) \\
\hline Parcelas x sub-parcelas & 74 & 5410,59 & & & \\
\hline Parcelas (F) & 14 & 124,94 & & & \\
\hline gl (bloques) & 2 & 0,13 & 0,064 & 0,386 & 4,46 \\
\hline gl (fluidos) & 4 & 123,48 & 30,870 & 185,815 & 3,84 \\
\hline Error (fluidos) & 8 & 1,33 & 0,166 & & \\
\hline gl (propiedades) & 4 & 5004,04 & 1251,009 & 3583,870 & 2,61 \\
\hline Fluidos x Propiedades & 16 & 267,66 & 16,728 & $\mathbf{4 7 , 9 2}$ & $\mathbf{1 , 9 0}$ \\
\hline Error (bloques) & 40 & 13,96 & 0,349 & &
\end{tabular}

La diferencia estadística obtenida conllevó a un estudio de diferencias medias significativas (DMS) de los fluidos y de sus propiedades. En la Tabla 7 se muestran las medias de los fluidos y de las propiedades para su comparación 
Tabla 7. Aplicación de las diferencias medias significativas, para comparar medias por tratamiento de los fluidos por propiedades

\begin{tabular}{lllllll}
\hline \multirow{2}{*}{ Fluido } & \multicolumn{7}{l}{ Propiedades } & \multicolumn{2}{l}{ Medias de fluido } \\
\cline { 2 - 7 } & VP & PC & EG 10s & EG 10min & Filtrado & \\
\hline Patrón & 34,67 & 6,33 & 8,67 & 15,67 & 6,33 & 14,33 \\
\hline FA & 25,33 & 6,00 & 4,33 & 7,33 & 10,67 & 10,73 \\
\hline FB & 26,33 & 5,67 & 4,67 & 9,67 & 10,73 & 11,41 \\
\hline FC & 25,67 & 5,67 & 4,33 & 9,67 & 10,63 & 11,19 \\
\hline FD & 26,33 & 6,33 & 4,67 & 9,33 & 10,33 & 11,40 \\
\hline Medias de propiedades & 27,67 & 6,00 & 5,33 & 10,33 & 9,74 & \\
\hline
\end{tabular}

Aplicadas las ecuaciones respectivas se obtuvo una DMS $=0,343$ para los fluidos y una $\mathrm{DMS}=0,436$ para las propiedades. En la Figura 4 se evidencia entonces que los fluidos FB, FC y FD presentan un comportamiento igual entre ellos, es decir las medias de sus propiedades se asemejan, pero difieren del fluido patrón (media por debajo) y a FA (media por encima), el cual también difiere del patrón. Este comportamiento corrobora la diferencia estadística obtenida en el estudio por parcelas divididas

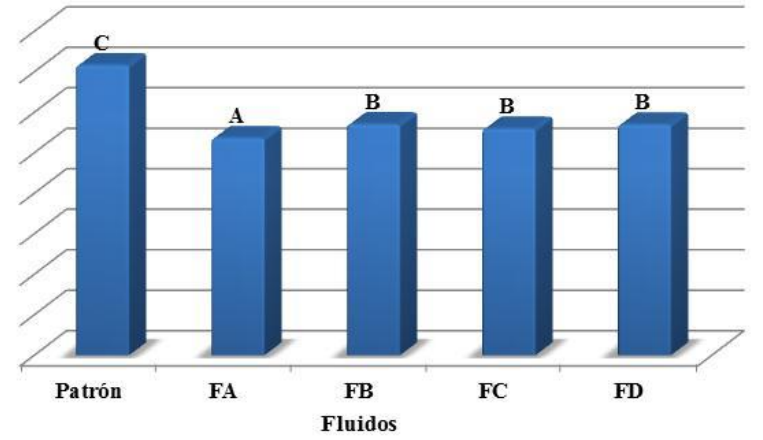

Figura 4. Comportamiento de los fluidos (Letras distintas expresan diferencias estadísticas significativas, $\mathrm{DSM}=0,343$ ).

Respecto a las propiedades en estudio, quedó evidenciado que la única propiedad donde no existió diferencia con el fluido patrón fue en el punto cedente, donde el FD se asemeja a él y los demás fluidos presentaron igual comportamiento entre ellos (Figura 5.b). En las propiedades Gel $10 \mathrm{~s}$ (Figura 5.c) y Filtrado (Figura 5.e) todos los fluidos con coque presentaron un mismo comportamiento sin diferencias estadística entre ellos. En la viscosidad plástica no presentaron diferencias significativas el FA con el FC y FB con FD (Figura 5.a) y en los geles a 10 min Fa difirió de FB, FC y FD que presentaron igual comportamiento (Figura 5.d). Lo anterior demuestra que la variación en la concentración de coque de petróleo de manera progresiva $(6 \mathrm{lb} / \mathrm{bl}, 8 \mathrm{lb} / \mathrm{bl}, 10 \mathrm{lb} / \mathrm{bl}$ y 12 $\mathrm{lb} / \mathrm{bl})$ no se refleja en un aumento o una disminución uniforme de las propiedades.

La metodología de Pinzón (2006) permitió transformar el coque en organófilico, no obstante hubo la necesidad de aumentar la concentración de amina cuaternaria para ello, aunque no todo se transformó posiblemente debido a la presencia de metales como Vanadio y Níquel (Sánchez et al., 2016). Los resultados obtenidos en las propiedades reológicas contrastan con los de Vega et al.; (2016) donde al aumentar la concentración de coque en fluidos base agua, el coque de petróleo se comportó como un modificador de reología. Si bien los valores obtenidos de las propiedades físicas de los fluidos difieren con el fluido patrón, estos no se deben descartar dado que los mismos están dentro de los rangos de valores aceptados para fluidos de perforación base aceite mostrados por (Correia et al., 2015). Además es importante señalar que el uso de un fluido o no depende de los rangos requeridos para una zona específica a perforar. 

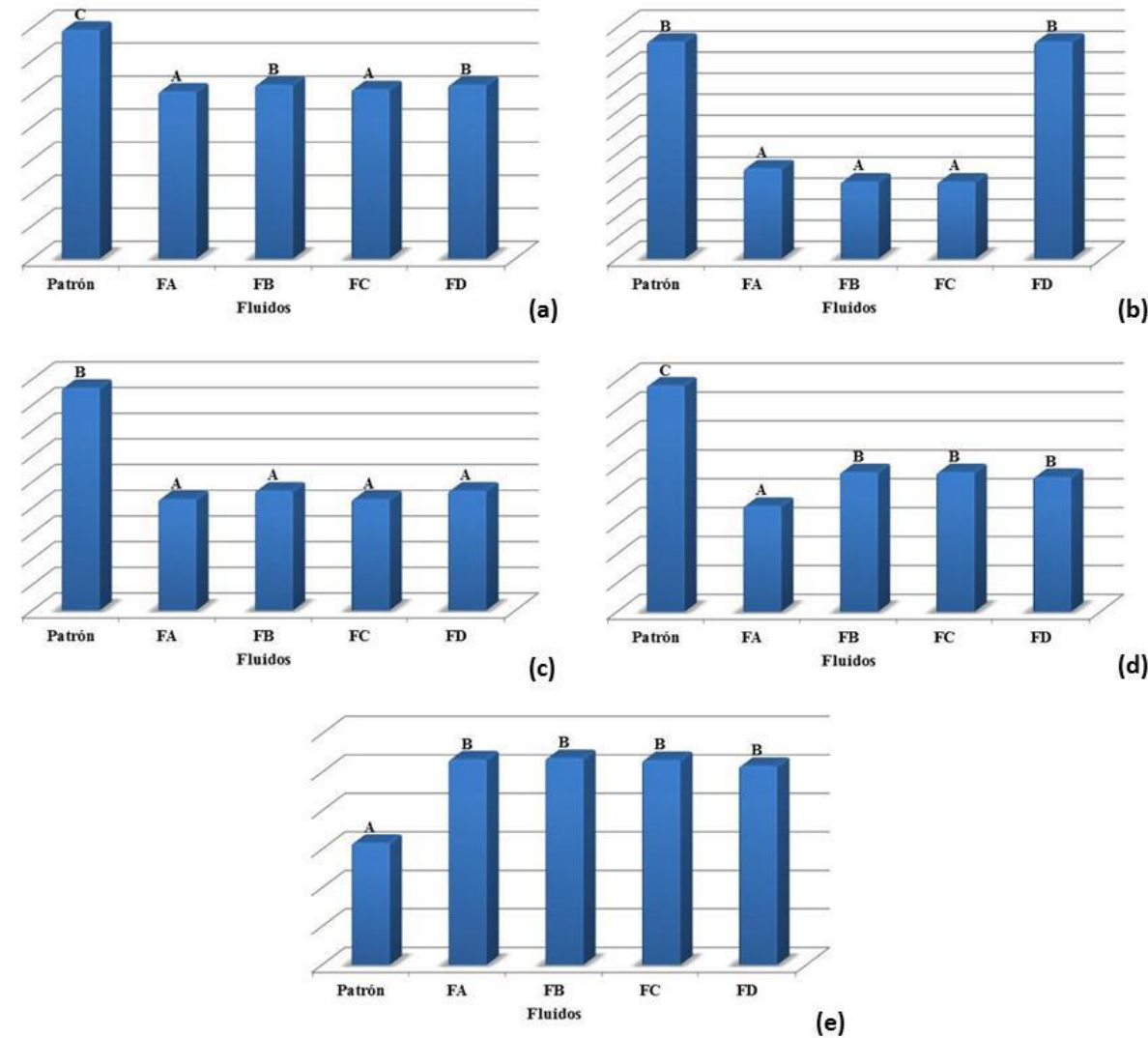

Figura 5. Diferencias medias significativas (DMS) de las propiedades a) Viscosidad plástica, b) Punto cedente, c) Gel $10 \mathrm{~s}$, d) Gel 10 min y e) filtrado, de los fluidos con el fluido patrón (DMS= 0,436 ) (letras distintas expresan diferencias estadísticas significativas)

\section{Conclusiones y Recomendaciones}

El Xileno es un buen disolvente para extraer el aceite de coque de petróleo mediante el método Soxhlet.

Se obtuvieron propiedades físicas de los fluidos aceptables en sus diferentes concentraciones para un fluido de perforación base aceite con lignito como controlador de filtrado.

A pesar de las diferencias estadísticas con el fluido patrón, quedó demostrado que se puede utilizar el coque de petróleo como controlador de filtrado bajo las condiciones en estudio.

Se hace necesario profundizar el estudio del coque de petróleo en fluidos de perforación base aceite como controlador de filtrado.

\section{Bibliografía}

API. (2003) API RP 13B-1, Recommended practice for field testing oil based fluids drilling fluids. Estados Unidos.

Ariza, E; Castro, A. y Gómez, J. (2013). Factibilidad del uso del almidón de achira como agente controlador de filtrado en lodos de perforación base agua. Rev.ion, 26(1), 63-71.

ASTM (2007). D3177-07, Standard test methods for sulfur in the analysis simple of coal and coke. Estados Unidos.

CIED-PDVSA. (2002). Fluidos de perforación. Caracas, Venezuela: CIED 
Correia, J., García, M., Godoy, G., \& Vega, R. (2015). Formulación de un fluido de perforaciónbase dieles de bajo impacto ambiental. Boletín de Geología, 37(2), 7582.

Da Silva, J.; Brandt, M. \& Ferreira, A. (2004). Coque de petróleo como energía primaria para una planta de generación de electricidad. Recuperado de: http://www.seeds.usp.br/pir/arquivos/congressos/CISEMALIE2005/cimaie/Venezuel a/VE\%20\%20DA\%20SILVA\%20\%20INELECTRA\%20\%20Coque\%20de\%20Petr\% F3leo\%20como\%20Energ\%EDa\%20Pr.pdf.

EPA (2015) Método 3540C. Soxhelt extraction, part of test methods for evaluating solids waste, physical/ chemical methods. Estados Unidos.

Faergestad, I y Strachan, C. (2014). Desarrollo de un fluido de alto rendimiento a base de aceite para perforación exploratoria. Oilfield Review, 26(1), 28-35.

Gómez, E., Andaverde, J., Santoyo, E. \& Urquiza, G. (2009). Determinación de la viscosidad y su incertidumbre en fluidos de perforación usados en la construcción de pozos geotérmicos: aplicación en el campo de Los Humeros, Puebla, México. Revista Mexicana de Ciencias Geológicas, 26(2), 516-529

Gorrin K, Basantas G, López J. \& Rodríguez A. (2011). Usos del coque de petróleo en los procesos productivos del sector Siderúrgico. Simposio llevado a cabo en las IX Jornadas de Investigación 2011. Puerto Ordaz, Venezuela

Katz, R. (2001). Efectos ambientales de la sustitución de carbón por petcoke en la generación eléctrica y procesos industriales. Revista Ambiente y Desarrollo, XVII(1), $22-29$.

Kräuter, R. \& Pérez, M (2003). Estudio preliminar del reciclaje de ácido en la desmetalización y desulfuración simultánea de coques de petróleo venezolanos vía microondas. Revista de la Facultad de Ingeniería UCV, 8(1), 73-81.

Little, T y Hills, J. (1998). Métodos estadísticos para la investigación en la agricultura. Ciudad de México, México: Trillas.

López, I. (2003). Fluidos de Perforación. Recuperado de: http:www.scribd.com/doc /25599222/Fluidos-de-Perforación.

Lowy, D.; Garrido, G; \& Melo, V. (2015). Predicción de variables operacionales en el proceso de Producción de Crudos por Segregación Gravitacional Asistida por Vapor (SAGD). Enfoque UTE, 6(2), 25 - 43.

MPPEUCT, 2012, Ejecutivo Nacional iniciará investigaciones para el aprovechamiento del Coque de Petróleo. Recuperado de: http://www.mppeuct.gob.ve/actualidad /noticias/ejecutivo-nacional-iniciara-investigaciones-para-el-aprovechamiento-delcoque-de

PDVSA (2006). Venezuela posee las reservas de crudo más grandes del mundo. Recuperado de: http://www.pdvsa.com/index.php?option=com_content\&view=article \&id=1501:2423\&catid=10\&ltemid=589\&lang=es

Pinzón, J. (2006). Viscosidad de suspensiones de una bentonita en estado natural y organofílica. Revista Colombiana de Química, 35(2), 225-232.

Prieto, A. (2007). Tecnología de los fluidos de perforación. Maracaibo, Venezuela: APOCA.

Samán Tecnología Integral en Petróleo (2010) ISGC-8.0.1-1-00, Manual de Calidad de fluidos de perforación base aceite. Maracaibo- Venezuela.

Sánchez, L., Campo, G. \& Cáceres, A. (2016). Algunos aportes en la extracción de vanadio, níquel y azufre en petrocoque. Revista Bases de la Ciencia,1(1),1-12.

Vega, R.; García, M.; Rivas, C.; Castillo, L.; Figueroa, Y. \& Hernández, O. (2016). Uso del coque del complejo petroquímico general de división José Antonio Anzoátegui como controlador de filtrado en fluidos base agua. Revista Ciencia e Ingeniería, 37(3), 137-146.

Wiliamson, D. (2013). Fundamento de los fluidos de perforación. Oilfield Review, 25(1), 67-69. 